Original Research Paper

\title{
Analytical Analysis of In-Band Crosstalk, Out-of-Band Crosstalk and GVD-Based Power Penalties in DWDM and TDM/DWDM-PONS
}

\author{
Ibrahim Mohamed and Mohammad Syuhaimi Ab-Rahman \\ Department of Electrical, Electronics and System Engineering, UKM University, Bangi, Malaysia
}

Article history

Received: 16-02-2014

Revised: 26-04-2015

Accepted: 04-05-2015

Corresponding Author: Ibrahim Mohamed

Department of Electrical,

Electronics and System

Engineering, UKM University,

Bangi, Malaysia

Email: engibrahim_2007@yahoo.com

\begin{abstract}
In addition to the ever-increasing demand for broader bandwidth per user, which results from the continuous development of new bandwidthhungry services and applications, the consequent upgrade from the currently deployed Time-Division Multiplexing Passive Optical Networks (TDMPONs) to Next-Generation Optical Access Networks (NG-OANs) has become inevitable. Different architectures for creating a NG-OAN have been proposed in the literature. Among those architectures, the DWDM and TDM/DWDM-Based OANs are very promising candidates. They were mainly proposed to exploit the large wavelength counts available in the fibre (its virtual unlimited bandwidth) to achieve a significant increase in the system capacity. Moreover, they allow coexistence in an open access environment among different network operators. In this study, we first analyze the impact of in-band crosstalk, out-of-band crosstalk to evaluate the performance of the Arrayed Waveguide Grating (AWG). The reason to focus on the AWG is due to this optical device is used almost in all DWDM and TDM/WDM-PONs. We then turn our attention to analyze the impact of group velocity dispersion GVD to estimate the maximum allowable bit rate for optical transmission without the need for using a Dispersion Management Technique (DMT) and/or a Forward Error Correction Technique (FECT). The analysis was performed using Matlab software (The Math works, Inc., Natick, MA, USA) and confirms that the in-band crosstalk has a stronger effect than the out-of-band crosstalk because its noise floor is reached at a lower crosstalk noise and with fewer crosstalk components. The in-band crosstalk noise should be kept below $-37 \mathrm{~dB}$ and $-34 \mathrm{~dB}$ to maintain a power penalty of less than $1 \mathrm{~dB}$ if 15 and 7 in-band crosstalk components are considered, respectively. The out-of-band crosstalk noise should be kept below $-20.3 \mathrm{~dB}$ and $-17.18 \mathrm{~dB}$ to maintain a power penalty of less than $1 \mathrm{~dB}$ if 240 and 56 out-of-band crosstalk components are considered, respectively. It was observed that the GVD noise floor is reached at a shorter fiber length as the bit rate increases and it was confirmed that a significant improvement in which the GVD noise floor is reached at longer fiber can be achieved if an externally modulated, small spectral-width source is used when a bit rate of $622 \mathrm{Mbps}, 1 \mathrm{Gps}$, or $2.5 \mathrm{Gbps}$ is used. However, a dispersion management technique becomes necessary if the bit rate increases to $10 \mathrm{Gbps}$ or more.
\end{abstract}

Keywords: Optical Access Networks, Optical Hybrid Schemes, FTTH, AWG, GVD, Crosstalk

\section{Introduction}

The history of investment in optical fibers for access networks started in the early nineties, when there was an anticipated growth of bandwidth demand. The first optical access network was the broadband passive optical network (BPON [G.983]), standardized by the International Telecommunications Union's Telecommunication Standardization Sector (ITU-T) in 1995. The BPON was followed by gigabit-class PONs 
(Ethernet PON [IEEE802.3ah] and Gigabit PON [G.984]), standardized in 2004 by the Institute of Electrical and Electronic Engineers (IEEE) and ITU-T, respectively. The downstream data rates offered by these PONs were $622 \mathrm{Mbps}, 1 \mathrm{Gbps}$ and $2.5 \mathrm{Gbps}$, respectively. To further increase the downstream data rate to $10 \mathrm{Gbps}$, IEEE and ITU-T ratified new standards (10G-EPON [IEEE802.3av] and XG-PON [ITU-T G.987]) in 2009 and 2010, respectively, for nextgeneration optical access networks. In all of the foregoing optical access networks, service was provided using Time-Division Multiplexing (TDM) technology in which a Point-to-Multipoint (P2MP) connection is established between one Optical Line Terminal (OLT) and several Optical Network Units (ONUs). Although TDM offers a cost-effective approach, its main drawback is its sharing-traffic nature, which poses a significant challenge towards future upgradeability. To overcome this limitation, different architectures have been proposed in the literature to create a next-generation optical access network (Grobe and Elbers, 2008; Kani, 2010; Kazovsky et al., 2011) based on either Wavelength-Division Multiplexing (WDM) or hybrid TDM/WDM technologies. In one of our studies (Syuhaimi and Mohamed, 2013), we proposed a hybrid TDM/DWDM-Based Long-Reach Optical Access Network (LR-OAN) scheme involving a two stage design. The design was based on a study of the characteristics of different optical components; including AWGs, Erbium-Doped Fiber Amplifiers (EDFAs) and Power Splitters (PSs) and the possibility of integrate these components together in one configuration to develop a hybrid TDM/DWDM scheme. While the AWG is used to realize the frequency re-use approach and handle upstream and downstream flow via the same input/output port through its properties of Wavelength Cyclic (WC) and Free Spectral Range (FSR), the EDFA is used to increase the capacity, extend the range and improve the design. Figure 1 shows a diagrammatic description of the design. In this study, we focus on providing analytical analyses concerning the first stage design. In these analyses, we first analyze the impact of in-band crosstalk and out-of-band crosstalk and their associated power penalties because they control the performance of the AWG in its application in the DWDM and/or TDM/DWDM optical networks as a multiplexer, demultiplexer, or router.

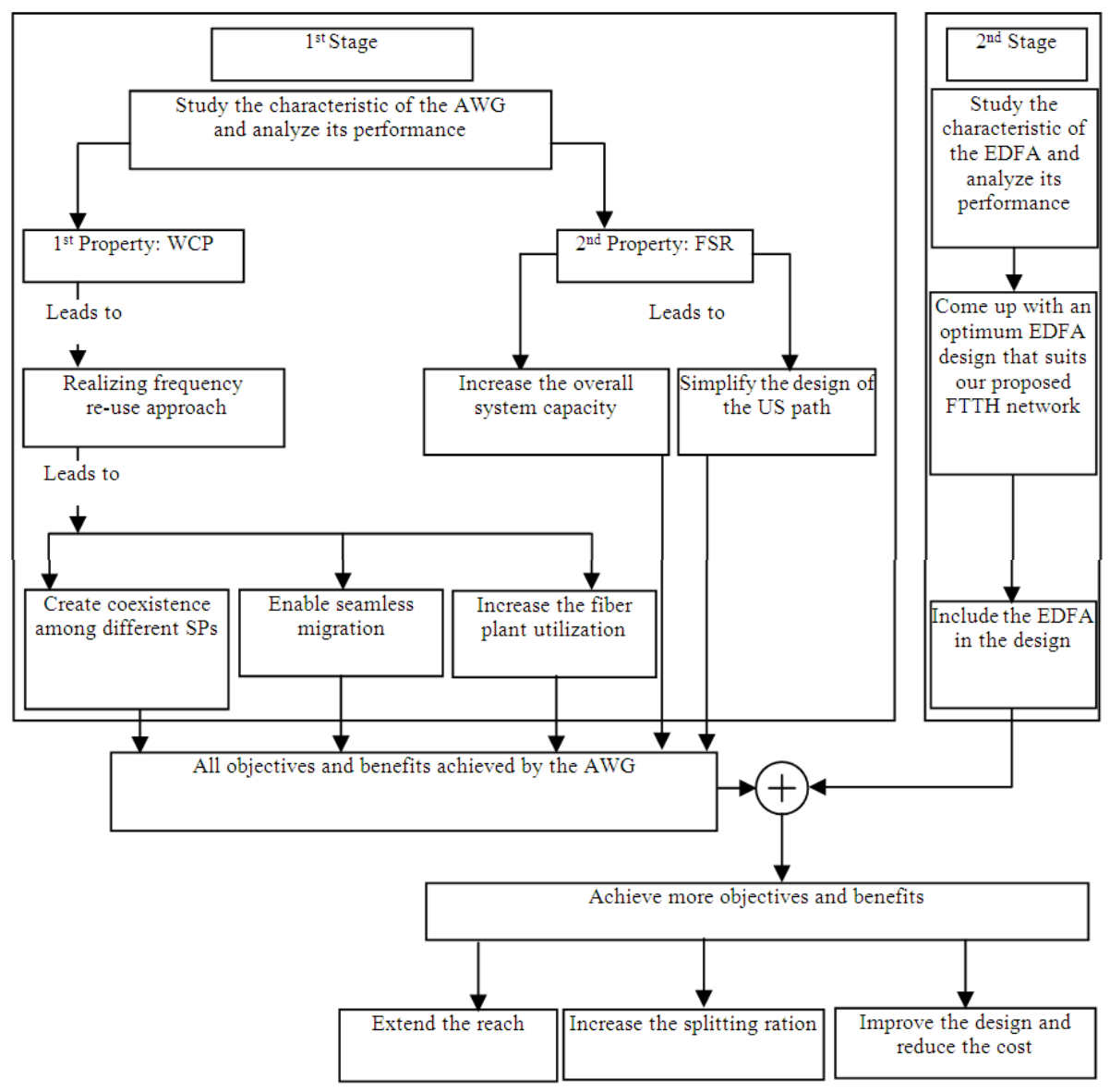

Fig. 1. Flow chart of the proposed two stage design 
We then turn our attention to provide an analytical analysis in terms of the GVD-based power penalty to evaluate the maximum allowable bit rate for optical transmission without the need for using any Dispersion Management Techniques (DMTs) and/or any forward Error Correction Techniques (FECTs). The power penalty $\left(P_{p}\right)$ is defined as the additional power required to maintain a specific Bit-Error Rate (BER) performance and is expressed in decibels as $P_{p}=10 \log _{10}\left[\frac{\text { Power_with_impairment }}{\text { Power_without_impairment }}\right]$. The paper is organized as follows: Section 2 provides an analysis of the impact of in-band crosstalk and outof-band crosstalk in an $\mathrm{N} \times \mathrm{N}$ AWGs and evaluates their associated power. Section 3 provides an analysis of the GVD effect and evaluates its associated power penalty. Section 4 is devoted for results and discussions. Section 5 concludes the paper with recommendation for future work.

\section{Crosstalk in an NXN AWG}

The Arrayed Waveguide Grating (AWG) is a promising optical device that can be deployed in dense WDM systems (Smit, 1988; Takahashi et al., 1993). The polarization dependence of this device can be eliminated using a mode converter scheme (Inoue et al., 1994), which also makes it attractive for use as a wavelength router in optical $\mathrm{N} \times \mathrm{N}$ interconnection systems. According to the Wavelength Cyclic Property (WCP) provided by the AWG (Kaneko et al., 1999), if $\mathrm{m}$ identical aggregations ( $\lambda 1$ to $\lambda n$ ) enter the $\mathrm{N}$ input ports of an $\mathrm{N} \times \mathrm{N}$ AWG, where $\mathrm{m}=\mathrm{n}=\mathrm{N}$, they will be distributed among its output ports with no overlap, i.e., each wavelength is included once in each input port and once in each output port. Examples of possible wavelength assignments of a $4 \times 4$ AWG are shown in Table 1 . In an $\mathrm{N} \times \mathrm{N}$ AWG, there are $N^{2}$ possible connections that can be established at the same time between the input ports and the output ports. Due to the narrow channel spacing in a dense WDM, any signal that propagates through one of these connections is affected by interference from the remaining $N^{2}-1$ connections.

Figure $2 \mathrm{a}$ and $\mathrm{b}$ schematically show 16 possible connections $\left(N^{2}\right)$ according to the wavelength assignment in Table 1, sample b where 15 interfering connections $\left(N^{2}-1\right)$ are divided into 3 interfering connections at the same wavelength $(N-1)$ and 12 interfering connections at different wavelengths $\left(N^{2}-N\right)$. This phenomenon is called linear crosstalk and is defined as the transfer of one channel's power to another channel. Here, the term linear crosstalk indicates that the power transferred in this case is due to an imperfection in the WDM components rather than the nonlinearity of optical channels.

While the effect of interfering connections at different wavelengths is also called out-of-band crosstalk is expected to be easily eliminated by suitable filtering at the receiver as this kind of interference has an incoherent nature and depends only on the power of the neighboring channel. The effect of interfering connections at the same wavelength due to beat noise caused by the $N$-1 crosstalk components, which is also called in-band crosstalk, is more severe because of its coherent nature, which causes the interference in this case to occur within the receiver bandwidth.

Table 1. Examples of possible wavelength assignment for $4 \times 4$ AWG

\begin{tabular}{|c|c|c|c|c|c|c|c|c|c|c|c|c|}
\hline Input/output ports & 1 & 2 & 3 & 4 & 1 & 2 & 3 & 4 & 1 & 2 & 3 & 4 \\
\hline 1 & $\lambda 1$ & $\lambda 2$ & $\lambda 3$ & $\lambda 4$ & $\lambda 1$ & $\lambda 4$ & $\lambda 3$ & $\lambda 2$ & $\lambda 1$ & $\lambda 2$ & $\lambda 3$ & $\lambda 4$ \\
\hline 2 & $\lambda 2$ & $\lambda 3$ & $\lambda 4$ & $\lambda 1$ & $\lambda 2$ & $\lambda 1$ & $\lambda 4$ & $\lambda 3$ & $\lambda 2$ & $\lambda 1$ & $\lambda 4$ & $\lambda 3$ \\
\hline 3 & $\lambda 3$ & $\lambda 4$ & $\lambda 1$ & $\lambda 2$ & $\lambda 3$ & $\lambda 2$ & $\lambda 1$ & $\lambda 4$ & $\lambda 3$ & $\lambda 4$ & $\lambda 1$ & $\lambda 2$ \\
\hline 4 & $\lambda 4$ & $\lambda 1$ & $\lambda 2$ & $\lambda 3$ & $\lambda 4$ & $\lambda 3$ & $\lambda 2$ & $\lambda 1$ & $\lambda 4$ & $\lambda 3$ & $\lambda 2$ & $\lambda 1$ \\
\hline Sample & $\mathrm{a}$ & & & & $\mathrm{b}$ & & & & $\mathrm{c}$ & & & \\
\hline
\end{tabular}

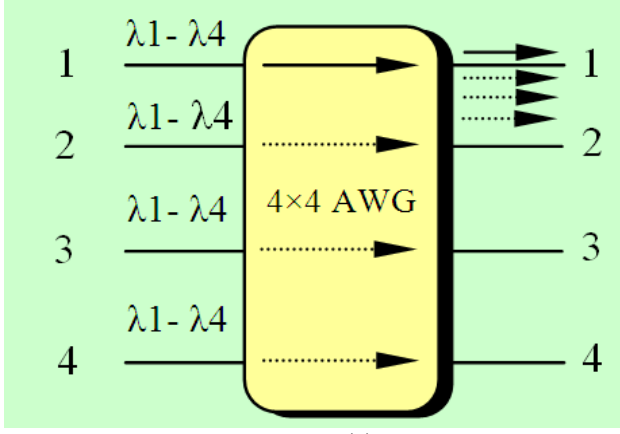

(a)

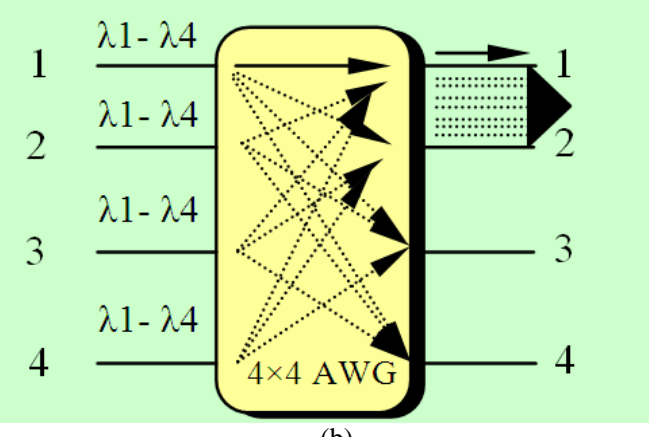

(b)

Fig. 2. (a) signal 3 same wavelength interfering components (N-1) (b) signal 12 different wavelength interfering components (N2-N) 
For example, according to the wavelength assignment shown in Table 1, sample $b$, if a portion of signal power carried by $\lambda 1$ between input/output ports (1/1) is leaked to output port 3 , its effect on the performance between input/output ports $(3 / 3)$ will be more detrimental than its effect on the performance between input/output ports $(3 / 1),(3 / 2)$ and $(3 / 4)$ because it has the same wavelength as the signal in this input/output port and therefore cannot be eliminated by filtering at the receiver. Both degrading effects can be evaluated by calculating the power penalty, which is defined as the increase in the average received power required to maintain a specific BER performance. In an $\mathrm{N} \times \mathrm{N}$ AWG, the field of the signal and its associated crosstalk components can be given by:

$$
E(t)=\sum_{i=o}^{N^{2}} E_{i} \sin \left(\omega t+\varphi_{i}(t)\right)
$$

Where:

$\omega=$ The optical carrier frequency and

$\varphi_{\mathrm{i}}(t)=$ The laser phase noise, which is equivalent to:

$$
\begin{aligned}
& E(t)=E_{o} \sin \left(\omega_{o} t+\varphi_{o}(t)\right) \\
& +\sum_{i=1}^{N-1} E_{i} \sin \left(\omega_{o} t+\varphi_{i}(t)\right)+\sum_{i=N}^{N^{2}-1} E_{i} \sin \left(\omega_{i} t+\varphi_{i}(t)\right)
\end{aligned}
$$

The 1 st and 2 nd terms of the right-hand side of (2) represent the signal and in-band crosstalk components, respectively. The 3rd term represents the out-of-band crosstalk components and is expected to have a less detrimental effect as its frequency components differ from the signal frequency.

\section{The Impact of In-Band Crosstalk}

In analyzing the impact of in-band crosstalk, we assume that the effect of the different frequency components is reduced by simply filtering them out using a suitable filtering process.

This assumption enables us to reduce Equation (2) to its 1 st and 2 nd terms, which leads to the following simplification:

$E(t)=E_{o} \sin \left(\omega_{o} t+\varphi_{o}(t)\right)+\sum_{i=1}^{N-1} E_{i} \sin \left(\omega_{o} t+\varphi_{i}(t)\right)$

At the receiver side, the primary current is given by:

$$
i_{p}=R P \equiv R E^{2}(t)
$$

where, $\mathrm{R}$ is the photodetector responsivity, measured in Amperes/Watt and $P$ is the average received power. Substituting (3) into (4) yields:

$$
\begin{aligned}
& i_{p}=R E_{o}^{2} \sin ^{2}\left(\omega_{o} t+\varphi_{o}(t)\right) \\
& +R \sum_{i=1}^{N-1} 2 E_{o} E_{i} \sin \left(\omega_{o} t+\varphi_{o}(t)\right)+R \sum_{i=1}^{N-1} E_{i}^{2} \sin ^{2}\left(\omega_{o} t+\varphi_{i}(t)\right)
\end{aligned}
$$

From (5), one can recognize the primary current $i_{p}$ by its three beat terms. The 1 st and 2 nd terms are signal-tosignal and signal-to-crosstalk beat terms, respectively. The 3rd term is a crosstalk-to-crosstalk beat term, which is small compared to the signal-to-crosstalk beat term and can be neglected in practice. The fraction of power added by the $N-1$ crosstalk components is referred to as the Relative Intensity Noise (RIN). From the central limit theorem, the probability distribution of the RIN becomes Gaussian as the number of crosstalk components increases (Agrawal, 2005). The mean square of the RIN can be obtained by adding the power of each in-band crosstalk component and dividing the sum by the signal power:

$$
\sigma_{R I N}^{2}=\frac{2 E_{o} \sum_{i=1}^{N-1} E_{i}}{E_{o}^{2}}=\frac{2 E_{o} E_{C}(N-1)^{-}}{E_{o}^{2}}=2 \varepsilon_{i n_{-} c}(N-1)
$$

The term $\varepsilon_{\text {in_c }}$ is the fraction of power added by one in-band crosstalk component, where an equal fraction of power is assumed by each in-band crosstalk component in (6). Considering that the signal-to-crosstalk beat occurs only when the crosstalk component is in the mark state (bit 1) and assuming that both the mark and absence states have equal probability densities $(\mathrm{p}(1)=\mathrm{p}(0)=$ $0.5)$, Equation (6) becomes:

$$
\sigma_{R I N}^{2}=\varepsilon_{i n_{-} c}(N-1)
$$

To evaluate the impact of in-band crosstalk, one must calculate the associated power penalty, which is defined as the increase in the average received power required to maintain a specific BER performance, hence counteracting the in-band crosstalk effect. To calculate the associated power penalty, one must (1) calculate the BER and the required primary current while considering only noise at the receiver, (2) calculate the required primary current that gives the same BER while considering noise at the receiver plus noise due to the crosstalk effect and (3) divide the primary current obtained in the second step by the primary current obtained in the first step. The BER determined without considering the effect of inband crosstalk in an Intensity Modulation and Direct Detection (IM-DD) scheme is given by:

$$
B E R=\frac{1}{4} \operatorname{erfc}\left(\frac{i_{p_{1}}-i_{D}}{\sigma_{1} \sqrt{2}}\right)+\frac{1}{4} \operatorname{erfc}\left(\frac{i_{D}-i_{p_{o}}}{\sigma_{o} \sqrt{2}}\right)
$$


where, $i_{p 1}, i_{p 0}$ are the average primary currents at bits 1 and 0 , respectively; $\sigma_{1}, \sigma_{0}$ are the receiver Root Mean Square (RMS) noise at bits 1 and 0 , respectively and $i_{D}$ is the decision threshold, given by $i_{D}=\frac{\sigma_{o} i_{p_{1}}-\sigma_{1} i_{p_{o}}}{\sigma_{o}+\sigma_{1}}$. For simplicity, we assume a special case in which the effective receiver noise is due only to thermal noise, which leads to equal noise contributions from both bit 1 and bit 0 , yielding $\sigma_{1}=\sigma_{o}=\sigma$. By considering this special case and assuming $i_{p o}=0$, the threshold current is simplified to $i_{D}=\frac{i_{p_{1}}}{2}$ and the BER in (8) becomes:

$B E R=\frac{1}{4} \operatorname{erfc}\left(\frac{i_{p_{1}}}{2 \sigma \sqrt{2}}\right)+\frac{1}{4} \operatorname{erfc}\left(\frac{i_{p_{1}}}{2 \sigma \sqrt{2}}\right)$

Which is equivalent to:

$B E R=\frac{1}{4} \operatorname{erfc}\left(\frac{Q}{\sqrt{2}}\right)+\frac{1}{4} \operatorname{erfc}\left(\frac{Q}{\sqrt{2}}\right)$

where, $Q=\frac{i_{p_{1}}}{2 \sigma}$ is referred to as the Q-Factor, which gives a measure of the performance (a BER of $10^{9}$ is obtained at $\mathrm{Q}=6$ ). By assuming a Gaussian distribution for the RIN, the total noise power determined by considering the effect of in-band crosstalk is given by $\sigma_{T}=\sqrt{\sigma^{2}+\sigma_{R I N}^{2} i_{p_{1}}^{2}}$. To evaluate the impact of in-band crosstalk on the BER, we consider its restricted effect at the mark state only and replace $\sigma$ in the first term of the right hand of (9) by $\sqrt{\sigma^{2}+\sigma_{R I N}^{2} i_{p_{1}}^{2}}$, which yields:

$B E R=\frac{1}{4} \operatorname{erfc}\left(\frac{i_{p_{1}}}{2 \sqrt{2} \sqrt{\sigma^{2}+\sigma_{R I N}^{2} i_{p_{1}}^{2}}}\right)+\frac{1}{4} \operatorname{erfc}\left(\frac{i_{p_{1}}}{2 \sigma \sqrt{2}}\right)$

To counteract the effect of in-band crosstalk, the primary current $i_{p 1}$ should be increased to $i_{p_{1}}^{\prime}$ so as to maintain a constant Q-Factor and hence a constant BER performance. The BER in (11) becomes:

$B E R=\frac{1}{4} \operatorname{erfc}\left(\frac{i_{p_{1}}^{\prime}}{2 \sqrt{2} \sqrt{\sigma^{2}+\sigma_{R I N}^{2} i_{p_{1}}^{2}}}\right)+\frac{1}{4} \operatorname{erfc}\left(\frac{i_{p_{1}}^{\prime}}{2 \sigma \sqrt{2}}\right)$

As mentioned above, the impact of in-band crosstalk is evaluated by calculating its associated power penalty. The power penalty in this case is given by:

$$
P_{P_{I N_{-} C}}=10 \log \left(\frac{i_{p_{1}}^{\prime}}{i_{p_{1}}}\right)
$$

The term $\frac{i_{p_{1}}^{\prime}}{i_{p_{1}}}$ is obtained using $Q=\frac{i_{p_{1}}}{2 \sigma}=\frac{i_{p_{1}}^{\prime}}{2 \sqrt{\sigma^{2}+\sigma_{R I N}^{2} i_{p_{1}}^{2}}}$ which leads to an in-band crosstalk power penalty of:

$P_{P_{I N_{-} C}}=-5 \log \left(1-4 \sigma_{R I N}^{2} Q^{2}\right)$

In $(7), \sigma_{R I N}^{2}=\varepsilon_{i n_{-} c}(N-1)$, which yields:

$P_{P_{N_{-} C}}=-5 \log \left(1-4 \varepsilon_{i n_{-} c}(N-1) Q^{2}\right)$

By considering $\mathrm{M}$ cascaded $\mathrm{N} \times \mathrm{N}$ AWGs, the power penalty due to the in-band crosstalk becomes:

$P_{P T_{I N_{-}} C}=-5 \log \left(1-4 M \varepsilon_{i n_{-} c}(N-1) Q^{2}\right)$

where, the term $4 M \varepsilon_{\text {in }_{-} c}(N-1)=\sigma_{\text {RIN }}^{2}$ TOTAL is the total relative intensity noise.

\section{The Impact of Out-of-Band Crosstalk}

Figure 3 schematically illustrates the effect of out-ofband crosstalk. This crosstalk originates from the transmissivity of the optical filters used in DWDM systems to pass a specific data bandwidth from neighboring channels. As stated above, the 3rd term in the right-hand side of Equation (2) represents the out-of-band crosstalk components. This term can be rewritten as:

$E_{3}(t)=\sum_{i=N}^{N^{2}-1} E_{i} \sin \left(\omega_{i} t+\varphi_{i}(t)\right)$

The total primary current associated with the out-ofband crosstalk components is given by:

$i_{p_{C-T O T A L}}=R E_{3}^{2}(t) \equiv R P_{C}\left(N^{2}-N\right) \equiv i_{p_{C}}\left(N^{2}-N\right)$

where, $P_{C}$ and $i_{P C}$ are the power and primary current associated with each out-of-band crosstalk component. The fraction of power added by each out-of-band crosstalk component is given by:

$\varepsilon_{\text {out }-c}=\frac{P_{c}}{E_{o}^{2}} \equiv \frac{P_{c}}{P_{s}} \equiv \frac{i_{p_{C}}}{i_{p_{S}}}$

where, $P_{s}$ and $i_{p s}$ are the power and the primary current associated with the intended signal. From (19), we obtain:

$i_{p_{C}}=\varepsilon_{\text {out }-c} i_{p_{S}}$ 
To evaluate the impact of out-of-band crosstalk, one must calculate the associated power penalty, which is defined as the increase in the average received power required to maintain a specific BER performance, thus counteracting this effect. To calculate the associated power penalty, one must follow the foregoing three steps as in the case of in-band crosstalk. Equation (10) can be used to calculate the BER without considering the effect of out-of-band crosstalk if an Intensity Modulation and Direct Detection (IM-DD) scheme is used. In this case:

$$
Q=\frac{i_{p_{S}}}{2 \sigma}
$$

By assuming that $i_{p c}$ has two values $\left(0\right.$ and $\left.i_{p c}\right)$, its average value can be given as $\frac{i_{p_{C}}+0}{2}=\frac{i_{p_{C}}}{2}$, which leads to its mean square $=\frac{i_{p_{C}}^{2}}{4}$. For the $\left(N^{2}-N\right)$ out-of-band crosstalk component, the mean square becomes $\left(N^{2}-N\right) \frac{i_{p_{C}}^{2}}{4}$. To calculate the BER while considering the out-of-band crosstalk effect, we consider its restricted effect on the mark state only and replace $\sigma$ in the first right-hand term of (10) with $\sqrt{\sigma+\frac{\left(N^{2}-N\right) i_{p_{C}}^{2}}{4}}$, which yields:

$$
Q=\frac{i_{p_{S}}}{\sqrt{\sigma^{2}+\frac{\left(N^{2}-N\right) i_{p_{C}}^{2}}{4}}}
$$

It is obvious that $\mathrm{Q}$ in (22) is smaller than its value in (21), which leads to a higher BER value. To maintain a constant $\mathrm{Q}$ and thus a constant BER performance, $i_{p s}$ in (22) should be increased to $i_{p_{S}}^{\prime}$, which leads to:

$$
Q=\frac{i_{p_{S}}}{2 \sigma}=\frac{i_{p_{S}}^{\prime}}{\sqrt{\sigma^{2}+\frac{\left(N^{2}-N\right) i_{p_{C}}^{2}}{4}}}
$$

From (23), the power penalty due to out-of-band crosstalk can be given as:

$P_{P_{\text {OUT }-C}}=10 \log \left(\frac{i_{p_{S}}^{\prime}}{i_{p_{S}}}\right)$

The term $\frac{i_{p_{S}}^{\prime}}{i_{p_{S}}}$ can be obtained from (23). Taking this into account and considering that the out-of-band crosstalk occurs only at the mark state and assuming that the mark and absence states have an equal probability density $(p(0)=p(1)=0.5)$, lead to an out-of-band power penalty as:

$$
P_{P_{\text {OUT_C }}}=-5 \log \left(1-0.5\left(N^{2}-N\right) Q^{2} \varepsilon_{\text {out }-c}^{2}\right)
$$

The power penalty in (25) was derived based on the assumption that an equal fraction of power is added by each out-of-band crosstalk component, i.e., a band-pass filter with equal transmissivity for each out-of-band crosstalk component is assumed to be employed. However, based on the schematic illustration of the transmission spectrum shown in Fig. 3, the filter transmissivity follows a Gaussian profile and becomes smaller as the out-of-band crosstalk component goes further from the selected signal on both sides. Based on this observation, the filter transmissivity to the $\left(N^{2}-N\right)$ component can be given as:

$$
T\left(N^{2}-N\right)=n \sum_{f=k, f \neq m}^{(N-1)} T(m, f)
$$

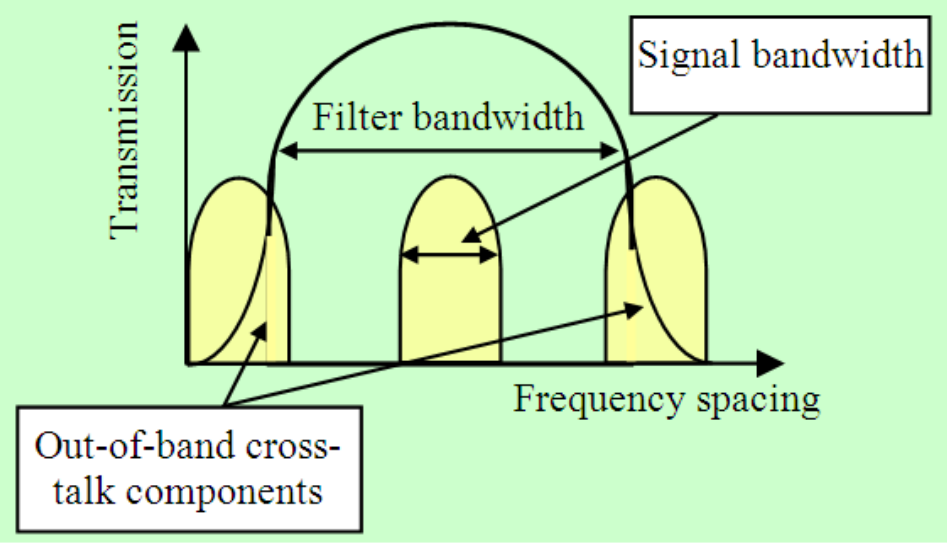

Fig. 3. Crosstalk due to different wavelength interfering channels (out-of-band crosstalk) 
where, $n=N$ refers to the number of out-of-band crosstalk frequency groups. For example for $4 \times 4$ AWG, we have 12 out-of-band crosstalk components $\left(N^{2}-N\right)$, which represent 4 groups of similar out-ofband crosstalk frequencies (12/3). The factors $f$ and $m$ are integers representing the out-of-band crosstalk component and the selected signal respectively, $f=k$, where $k$ is integer and $f \neq m$. for example, for $2 \times 2$ AWG, the filter transmissivity to the out-of-band crosstalk component when assuming the selected signal $m=0$ is given by:

$$
T\left(N^{2}-N\right)=n \sum_{f=k, f \neq m}^{(N-1)} T(m, f)=2 \sum_{f=1, f \neq 0}^{(1)} T(0, f)=2 T(0,1)
$$

Equation (27) can be rewritten in such a way as to follow the filter Gaussian profile:

$$
T\left(N^{2}-N\right)=n \sum_{f=k, f \neq m}^{(N-1)} e^{-(a f-m)^{2} / 2 f_{o}^{2}}
$$

where, $a$ is the frequency spacing and $f_{o}$ is a factor governs the filter spectrum width and related to the full width half maximum by $f_{o} \approx \mathrm{FWHM} / 1.665$. For example, for a Gaussian profile optical filter with $40 \mathrm{GHz}$ bandwidth, employed at $50 \mathrm{GHz}$ frequency spacing, the transmissivity to $N^{2}-N$ out-of-band crosstalk components can be given as:

$$
T\left(N^{2}-N\right)=n \sum_{f=k, f \neq m}^{(N-1)} e^{-(50 f-m)^{2} / 2(40 / 1.665)^{2}}
$$

Which shows a different contribution by each outof-band crosstalk frequency, leading to smaller out-ofband crosstalk impact.

\section{The Impact of GVD}

Although intermodal dispersion is not an issue in Single Mode Fibers (SMFs) because the energy of an input pulse to an SMF is transferred by only one mode (the fundamental mode), a phenomenon referred to as chromatic dispersion, also called group velocity dispersion GVD, leads to the broadening of pulses as they propagate through an SMF. This result arises from the dependency of the group velocity associated with the fundamental mode on the frequency. Because an optical pulse occupies a finite frequency span, its width will be broadened as it propagates through an SMF due to the GVD effect. In an optical communication system, GVD affects the receiver performance in two ways. First, the pulse peak power decreases as the optical pulse broadens, leading to a consequent reduction in the pulse energy within the bit slot and a reduction in the SNR at the receiver decision circuit. Because the SNR should remain constant to maintain desirable BER performance in a digital optical communication system, an increase in the average power at the receiver is required. This increase in the average power is referred to as the power penalty and is imposed to compensate for energy reduction within the bit slot at the receiver decision circuit. Second, as an optical pulse broadens, a portion of its energy spreads out of its allocated bit slot, which leads to the Inter-Symbol Interference (ISI) effect and degrades the receiver performance. To reduce the ISI effect, the receiver is designed such that the input signal to its decision circuit corresponds to the transfer function of a raised cosine filter. In a practical optical communication system, an optical pulse does not have an exact rectangular shape; a Gaussian (bell-shaped) pulse is commonly used as an approximation, allowing the signal pulse to be described by its average (mean), mean square (variance) and standard deviation (root mean square). To estimate the power penalty imposed by the GVD effect, consider the input Gaussian pulse within the bit slot $T_{b}$ shown in Fig. 4.

The RMS of the given input signal is $\sigma_{o}$, given by $\sigma_{o}=\frac{T_{o}}{\sqrt{2}}$, where $T_{o}$ controls the pulse width and is related to the pulse full width at half maximum by $T_{F W H M}$ $\approx 1.665 T_{o}$. Using approximately Gaussian statistics and accounting for both the data and source spectrum widths, the variance of the output broadened pulse $\sigma^{2}$ is given by:
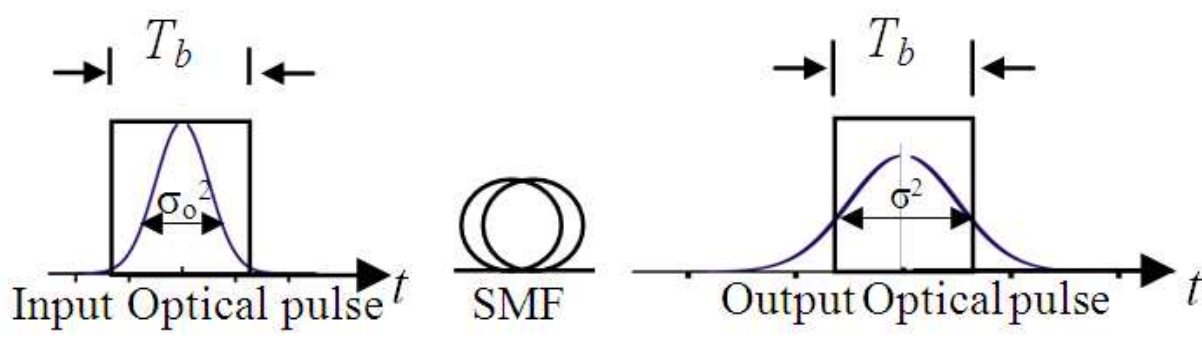

Fig. 4. Input optical Gassian pulse and its associated output broadened Gaussian pulse 


$$
\begin{aligned}
& \sigma^{2}=\sigma_{o}^{2}\left(1+\frac{C \beta_{2}}{2 \sigma_{o}^{2}}\right) \\
& +\sigma_{o}^{2}\left(1+V_{\omega}^{2}\right)\left(\frac{\beta_{2} L}{2 \sigma_{o}^{2}}\right)^{2}+\sigma_{o}^{2}\left(1+C^{2}+V_{\omega}^{2}\right)\left(\frac{\beta_{3} L}{4 \sqrt{2} \sigma_{o}^{3}}\right)
\end{aligned}
$$

This variance is equivalent to the sum of the individual variances of the input pulse and its associated dispersion $\left(\sigma^{2}=\sigma_{o}^{2}+\sigma_{D}^{2}\right)$, where $C$ governs the frequency chirp imposed on the pulse, $\beta_{2}$ and $\beta_{3}$ represent the second-and third-order derivatives of the propagation constant $\beta$, respectively and $V_{\omega}=2 \sigma_{\omega} \sigma_{\mathrm{o}}$, where $\sigma_{\omega}$ represents the RMS of the source spectrum width (Agrawal, 2010). While many cases can be presented by observing Equation (28), we only consider the three cases that fit our system design. In these cases, the term $\beta_{3}$ is assumed to be zero as the system is designed to work in $C$-band (away from zero dispersion). In the first case, a directly modulated large spectrum-width source is considered. For example, a directly modulated FP laser is used. In the second case, a directly modulated small spectrum-width source is considered. For example, a directly modulated DFB laser is used. In the third case, an externally modulated small spectral-width source is considered. For example, an externally modulated DFB laser is utilized.

\section{The First Cased (Directly Modulated Large Spectrum-Width Source is Considered)}

In this case, $V_{\omega}$ in (28) is assumed to be $>1$, which leads to $\left(1+V_{\omega}^{2}\right) \approx V_{\omega}^{2}$.To simplify the analysis, we consider a chirp-less input Gaussian with $\mathrm{C}=0$. Considering the above yields the following:

$$
\sigma^{2}=\sigma_{O}^{2}\left[1+\left(V_{\omega}^{2}\right)\left(\frac{\beta_{2} L}{2 \sigma_{O}^{2}}\right)^{2}\right]
$$

Substituting $V_{\omega}=2 \sigma_{\omega} \sigma_{\mathrm{o}}$ yields:

$$
\sigma^{2}=\sigma_{o}^{2}+\left(D L \sigma_{\lambda}\right)^{2} \equiv \sigma_{o}^{2}+\sigma_{D}^{2}
$$

where, $\beta_{2} \sigma_{\omega}=D \sigma_{\lambda}$. $D$ is a dispersion parameter measured in $\mathrm{ps} /(\mathrm{nm} \times \mathrm{km})$ and $\sigma_{\lambda}$ is the RMS of the source spectrum width in wavelength units. The RMS of the output broadened pulse is simply obtained by taking the square root of its variance and is given by $\sigma=\left(\sigma_{o}^{2}+\sigma_{D}^{2}\right)^{0.5}$, where $\sigma_{D}$ is the RMS dispersion, which is related to the dispersion parameter, the fiber length and the RMS of the source spectrum by $\sigma_{D}=|D| L \sigma_{\lambda}$. Division of the RMS of the output broadened pulse by the RMS of the input pulse gives a normalized estimation of the pulse broadening.
This unit-less value is called the broadening factor $B_{F}$ and can be derived as follows:

$$
\left[\frac{\sigma^{2}}{\sigma_{o}^{2}}\right]=\left[\frac{\sigma}{\sigma_{o}}\right]^{2}=\left[\frac{\left(\sigma_{o}^{2}+\sigma_{D}^{2}\right)}{\sigma_{o}^{2}}\right]=\left[1+\left(\frac{\sigma_{D}}{\sigma_{o}}\right)^{2}\right]=\left[1+\left(\frac{D L \sigma_{\lambda}}{\sigma_{o}}\right)^{2}\right]
$$

Taking the square root of $\left[\frac{\sigma}{\sigma_{o}}\right]^{2}$ yields:

$$
B_{F}=\left[\frac{\sigma}{\sigma_{o}}\right]=\left[1+\left(\frac{D L \sigma_{\lambda}}{\sigma_{o}}\right)^{2}\right]^{\frac{1}{2}}
$$

We relate the broadening factor $B_{F}$ to the bit rate $B_{T}$ by using the criterion $\sigma B_{T} \leq \frac{1}{4}$, which guarantees that $95 \%$ of the pulse energy can be kept within the allocated bit slot and leads to the maximum bit rate $B_{T}=\frac{1}{4 \sigma}$. Considering this criterion and considering that $\sigma_{o}=\frac{\sigma}{B_{F}}$, Equation (30) becomes $\quad B_{F}=\left[1+\left(\frac{D L \sigma_{\lambda} B_{F}}{\sigma}\right)^{2}\right]^{\frac{1}{2}}$ given that $\sigma=\frac{1}{4 B_{T}}$, yielding $B_{F}=\left[1+\left(D L \sigma_{\lambda} B_{T} B_{F}\right)^{2}\right]^{\frac{1}{2}}$. Solving this equation for $B_{F}$ yields:

$$
B_{F}=\frac{1}{\left[1-\left(4 D L \sigma_{\lambda} B_{T}\right)^{2}\right]^{\frac{1}{2}}}
$$

Because the broadened optical pulse has the same energy as the input pulse, an associated decrease in the peak power due to the GVD is expected by the same factor $B_{F}$, which leads to a consequent power penalty in $\mathrm{dB}$ to maintain a constant energy within the bit slot $\mathrm{T}_{\mathrm{b}}$ and thus a constant SNR. The power penalty in $\mathrm{dB}$ due to the GVD effect is defined as the increase in average power required to maintain a specific BER, given by:

$$
P_{P_{G V D}}=10 \log _{10} B_{F}=-5 \log \left[1-\left(4 D L \sigma_{\lambda} B_{T}\right)^{2}\right]
$$

\section{The Second Cased (Directly Modulated Small Spectrum-Width Source is Considered)}

In this case, the term $V_{\omega}=2 \sigma_{\omega} \sigma_{0}$ in (29) is replaced by $V_{\omega}=2 \sigma_{\mathrm{S} \omega} \sigma_{\mathrm{o}}$, which yields:

$$
\sigma^{2}=\sigma_{o}^{2}+\left(D L \sigma_{s}\right)^{2} \equiv \sigma_{o}^{2}+\sigma_{D}^{2}
$$


where, $\beta_{2} \sigma_{\mathrm{S} \omega}=D \sigma_{\mathrm{S}}$. $\mathrm{D}$ is the dispersion parameter measured in $\mathrm{ps} /(\mathrm{nm} \times \mathrm{km})$ and $\sigma_{\mathrm{s}}$ is the RMS of the signal spectrum width in wavelength units. Following the same procedures employed in the first case, the power penalty due to the GVD effect in this case is given as:

$$
P_{P_{G V D}}=-5 \log _{10}\left[1-\left(4 D L \sigma_{s} B_{T}\right)^{2}\right]
$$

\section{The Third Cased (An Externally Modulated Small Spectral-Width Source is Considered)}

In this case, $V_{\omega}$ in (28) is assumed to be $<<1$, which leads to $\left(1+V_{\omega}^{2}\right) \approx 1$. To simplify the analysis, we consider a chirp-less input Gaussian with $C=0$. Considering the above yields:

$$
\sigma^{2}=\sigma_{O}^{2}\left[1+\left(\frac{\beta_{2} L}{2 \sigma_{O}^{2}}\right)^{2}\right]
$$

$$
\text { From (34), } \sigma^{2}=\left[\sigma_{o}^{2}+\sigma_{o}^{2}\left(\frac{\beta_{2} L}{2 \sigma_{o}^{2}}\right)^{2}\right], \quad \text { which is }
$$
equivalent to $\sigma^{2}=\sigma_{o}^{2}+\sigma_{D}^{2}$. In this case, the dispersioninduced broadening depends on the initial width $\sigma_{o}^{2}$.

It is found that $\sigma$ is a minimum at $\sigma_{o}=\left(\frac{\left|\beta_{2}\right| L}{2}\right)^{\frac{1}{2}}$ and is given by $\sigma=\left(\left|\beta_{2}\right| L\right)^{\frac{1}{2}}$ (Agrawal, 2010). Using the criterion of $\sigma B_{T} \leq \frac{1}{4}$ and following the same procedures employed in the first and second cases, the power penalty due to the GVD effect in this case can be given as:

$P_{P_{G V D}}=-5 \log _{10}\left[1-\left(4 B_{T} \sqrt{\left|\beta_{2} L\right|}\right)^{2}\right]$

\section{Results}

This section is divided into two subsections. In the first subsection, results concerning the impact of inband crosstalk and out-of-band crosstalk and their associated power penalties are discussed, whereas, in the second subsection, results concerning the impact of GVD and its associated power penalty are discussed. These results are based on mathematical derivations that have been performed in the previous sections and obtained by using Matlab software (The Mathworks, Inc., Natick, MA, USA).

\section{Power Penalty Due to Crosstalk in an NXN AWG}

The results in this section are based on the mathematical derivations that have been performed in subsections 2.1 and 2.2 where the effects of in-band crosstalk and out-of-band crosstalk in an $\mathrm{N} \times \mathrm{N}$ AWG were evaluated, respectively.

\section{Power Penalty Due To in-Band Crosstalk in an $N \times N A W G$}

The power penalty due to in-band crosstalk in $\mathrm{N} \times \mathrm{N}$ AWGs is evaluated by considering Equations (15) and (16). In (15), an $\mathrm{N} \times \mathrm{N}$ AWG was considered, while in (16), a number of cascaded $\mathrm{N} \times \mathrm{N}$ AWGs were considered. Figure 5 shows the graphical representation of (15). It represents the power penalty versus the inband crosstalk for different numbers of in-band crosstalk components (7 and 15 in-band crosstalk components). It is obvious that the power penalty increases in each case as the in-band crosstalk noise increases until the noise reaches the point of the in-band crosstalk floor, where the penalty tends to infinity.

However, the in-band crosstalk floor is reached at a smaller in-band crosstalk noise level as the number of inband crosstalk components increases. Figure 6 shows the power penalty versus the in-band crosstalk for 15 inband crosstalk components and for different numbers of cascaded AWGs (2, 4 and 8 cascaded AWGs). Similarly, the power penalty increases in each case as the in-band crosstalk noise increases until the noise reaches the point of the in-band crosstalk floor, where the penalty tends to infinity. However, the in-band crosstalk floor is reached at a smaller in-band crosstalk noise level as the number of cascaded AWGs increases.

\section{Power Penalty Due to Out-of-Band Crosstalk in $N \times N$ AWGs}

The power penalty due to in-band crosstalk in $\mathrm{N} \times \mathrm{N}$ AWGs is evaluated by considering Equations (25) and (27). In (25), equal fractions of noise power were considered, whereas in (27), unequal fractions of noise power were considered.

Figure 7 shows the graphical representation of (25). It represents the power penalty versus the out-ofband crosstalk for different numbers of out-of-band crosstalk components (56 and 240 out-of-band crosstalk components).

It is obvious that the power penalty increases in each case as the out-of-band crosstalk noise increases until the noise reaches the point of the out-of-band crosstalk floor, where the penalty tends to infinity. However, the out-ofband crosstalk floor is reached at a smaller out-of-band crosstalk noise level as the number of out-of-band crosstalk components increases. 


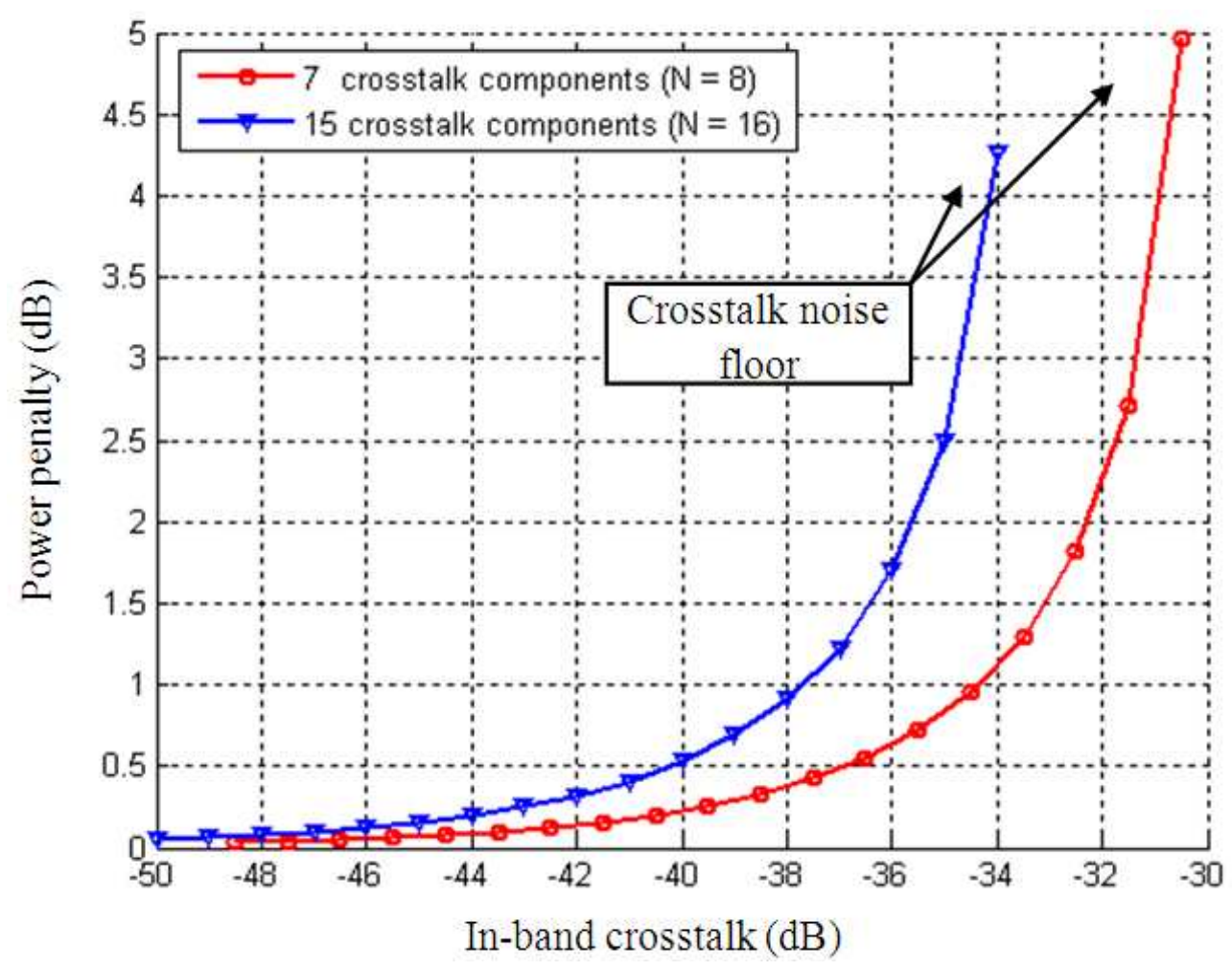

Fig. 5. Power penalty versus in-band crosstalk at different in-band crosstalk components

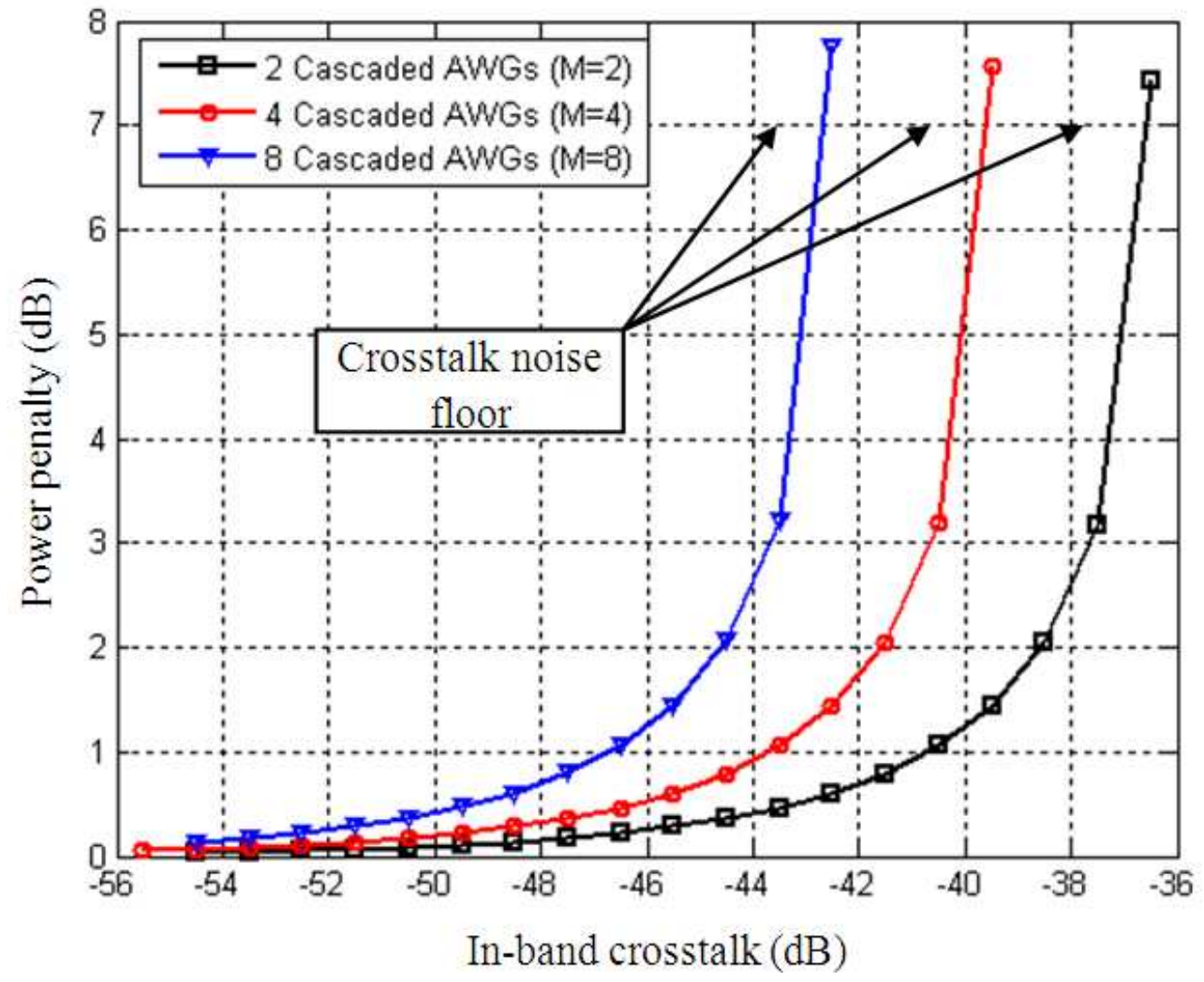

Fig. 6. Power penalty versus in-band crosstalk at 15 in-band crosstalk components and different number of cascaded AWGs crosstalk 


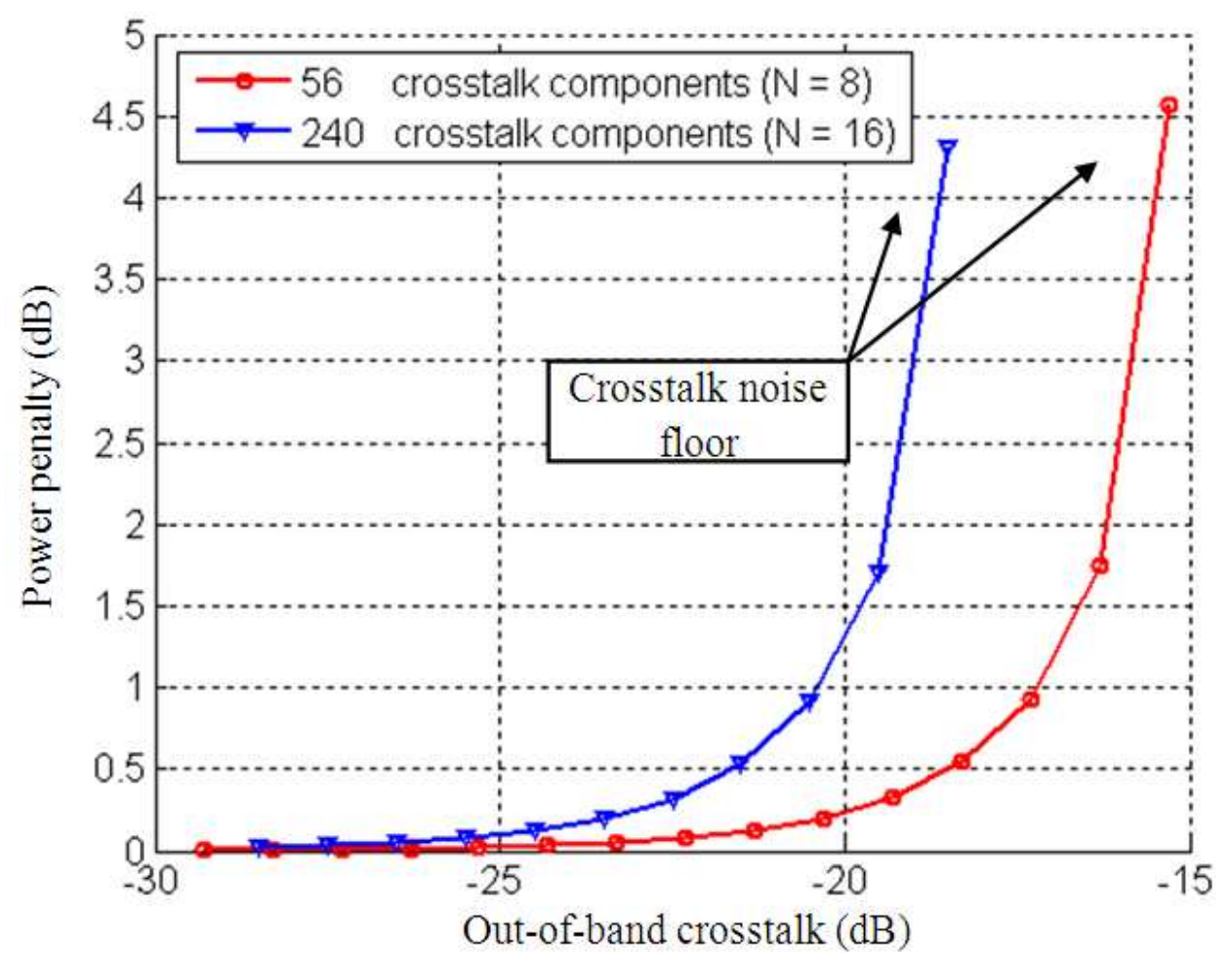

Fig. 7. Power penalty versus out-of-band crosstalk at different out-of-band crosstalk components

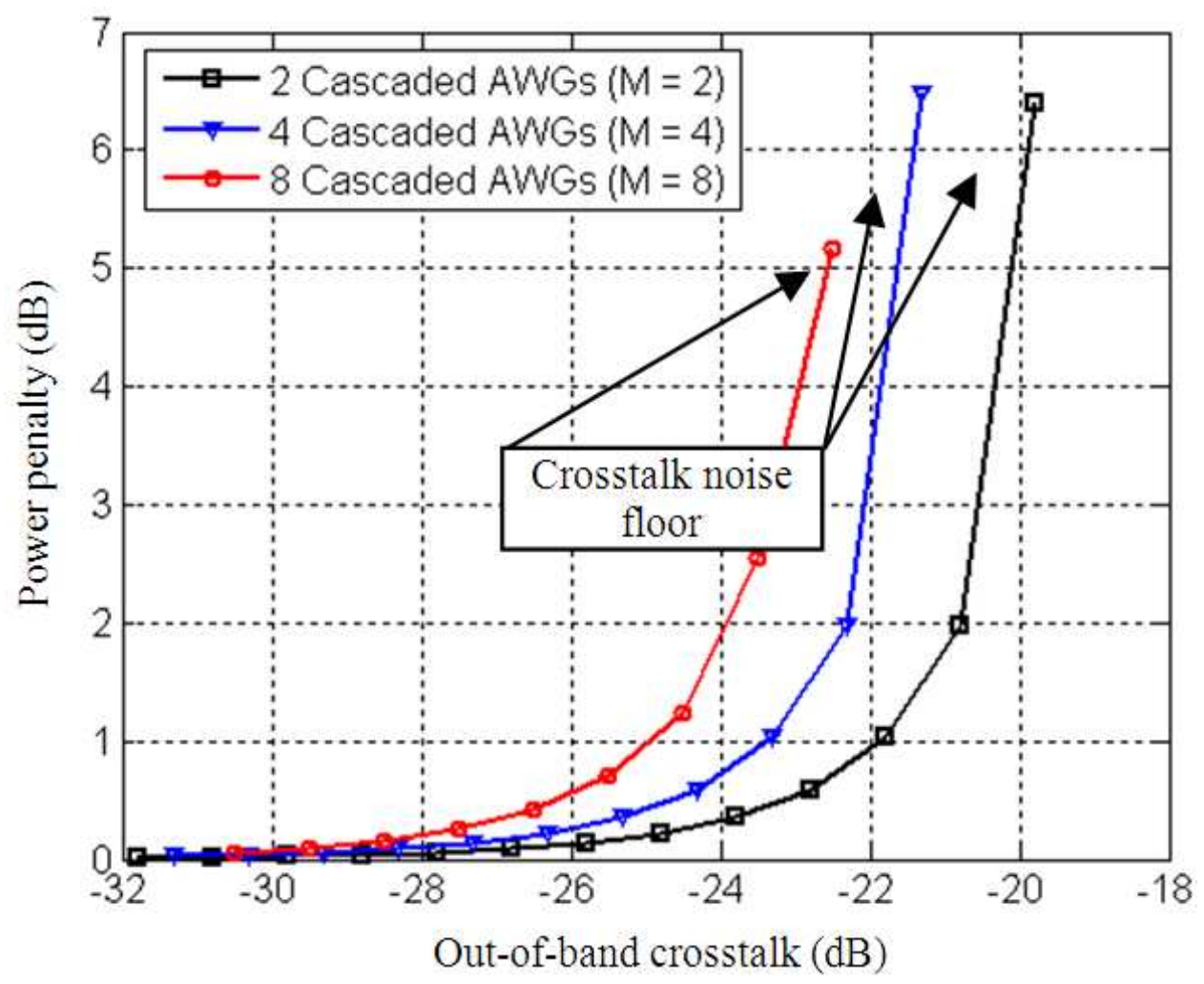

Fig. 8. Power penalty versus out-of-band crosstalk at 256 out-of-band crosstalk components and different number of cascaded AWGs 


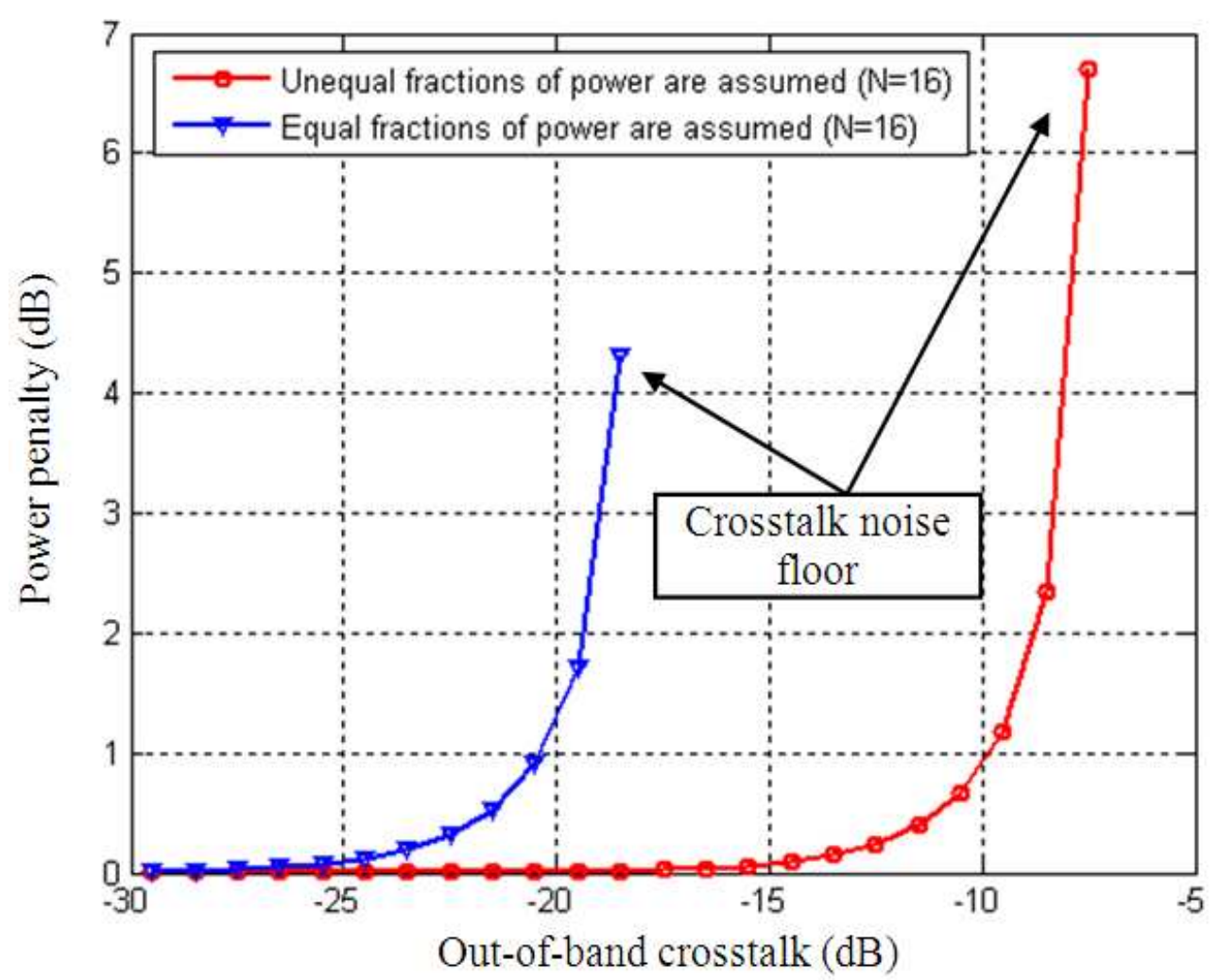

Fig. 9. Power penalty versus out-of-band crosstalk noise, equal and unequal fractions of noise power are considered

Figure 8 shows the power penalty versus the out-ofband crosstalk for 240 out-of-band crosstalk components and for different numbers of cascaded AWGs (2, 4 and 8 cascaded AWGs). Similarly, the power penalty increases in each case as the out-of-band crosstalk noise increases until the noise reaches the point of the out-of-band crosstalk floor, where the penalty tends to infinity. However, the out-of-band crosstalk floor is reached at a smaller out-of-band crosstalk noise level as the number of cascaded AWGs increases. Compared with the in-band crosstalk, we find that the out-of-band crosstalk has a weaker effect because its noise floor is reached at a higher out-ofband crosstalk noise level and for a larger number of out-of-band crosstalk components.

Figure 9 shows a graphical representation of Equations (25) and (27), where equal and unequal fractions of noise power are considered, respectively.

It is obvious that the point of the out-of-band crosstalk floor is reached at a higher out-of-band crosstalk noise level for the case in which a different fraction of noise power is considered by each out-ofband frequency.

\section{Power Penalty Due to GVD}

The results in this section are based on the mathematical derivations that have been performed in subsections 3.1, 3.2 and 3.3 where a directly modulated large spectrum-width source, a directly modulated small spectrum-width source and an externally modulated small spectral-width source were considered, respectively.

\section{Power Penalty Due to GVD (Directly Modulated Large Spectrum-Width Source is Considered)}

In this case, the power penalty due to GVD is evaluated by considering equation (32). Figure 10 shows the graphical representation of (32). It represents the power penalty Vs. the fiber length for different bit rates (622 Mbps, $1 \mathrm{Gbps}$ and $2.5 \mathrm{Gbps})$. It is clear that the power penalty increases in each case as the fiber length increases until the GVD reaches the point of the GVD noise floor, where the penalty tends to infinity.

The GVD noise floor is reached at a shorter fiber length as the bit rate increases. i.e., the fiber lengths that can be used if a penalty of $1 \mathrm{~dB}$ is allowed are $7.64 \mathrm{~km}, 4.75 \mathrm{~km}$ and $1.9 \mathrm{~km}$ at $622 \mathrm{Mpbs}, 1 \mathrm{Gpbs}$ and $2.5 \mathrm{Gbps}$, respectively. However, these fiber lengths confirm the invalidity of using the directly modulated large spectrum-width source as a transmitter in any optical access network where a minimum length of 20 $\mathrm{km}$ was specified. 


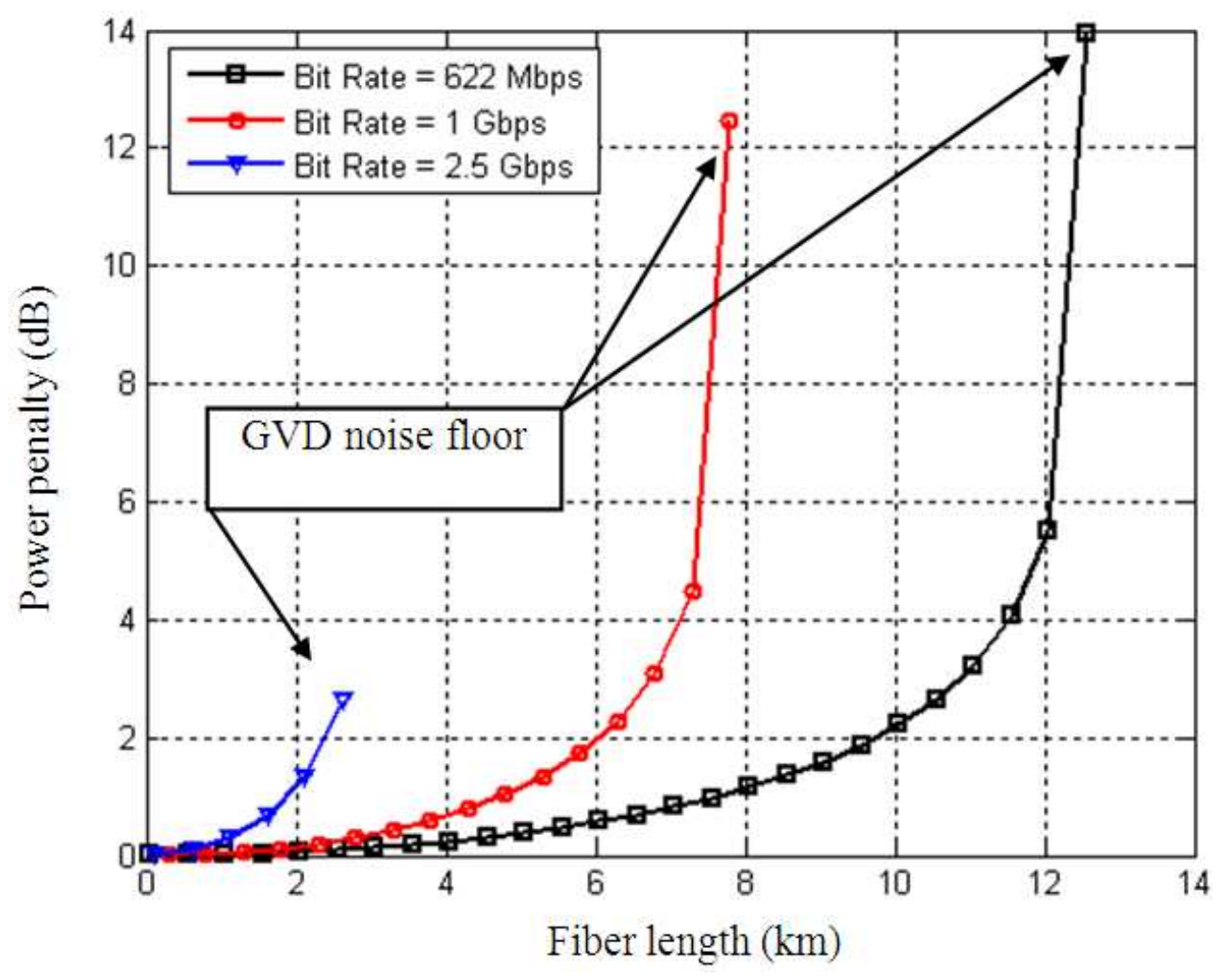

Fig. 10. Power penalty due to GVD versus fiber length at different bit rates (first case)

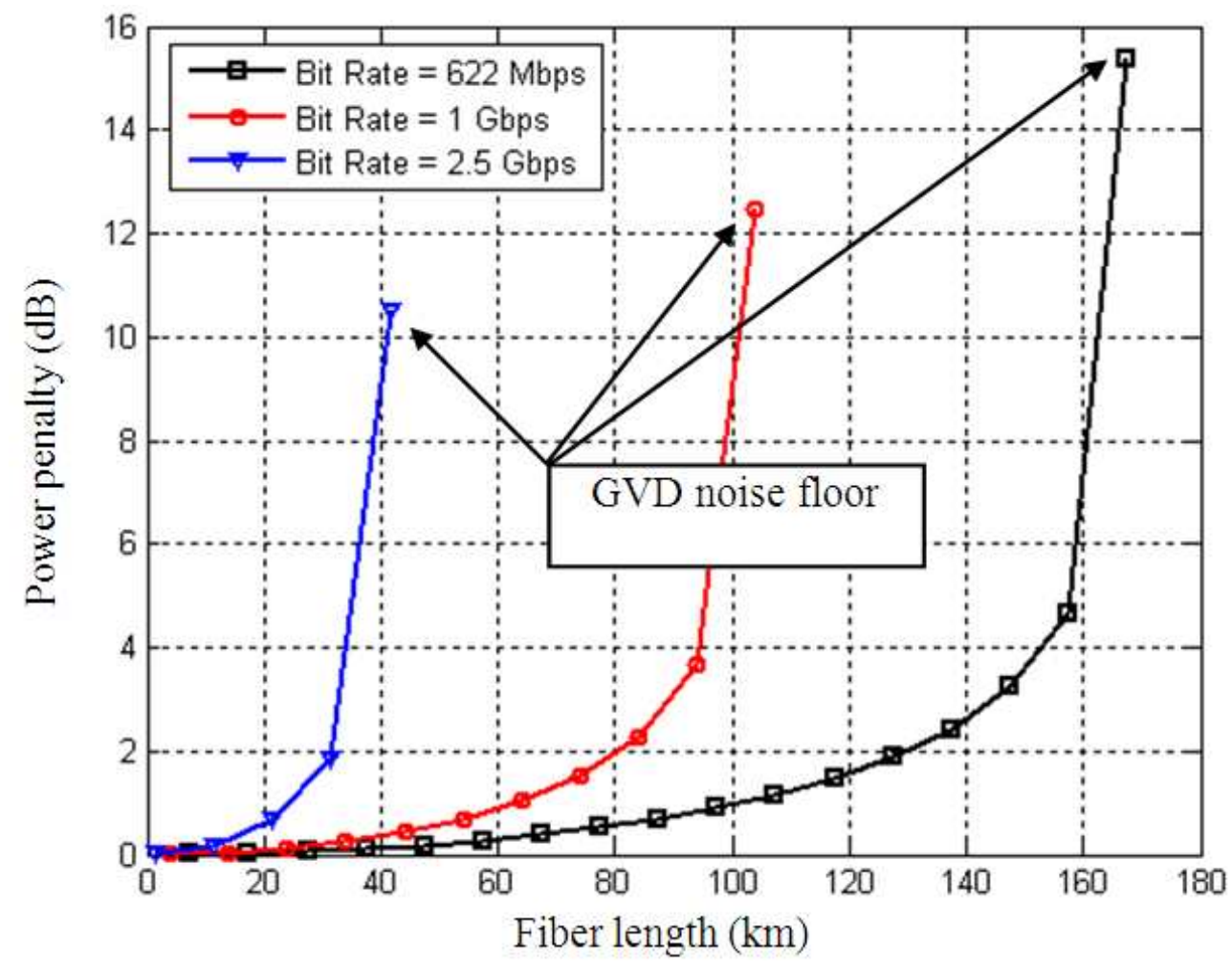

Fig. 11. Power penalty due to GVD versus fiber length at different bit rates (second case) 
Power Penalty Due to GVD (Directly Modulated Small Spectrum-Width Source is Considered)

In this case, the power penalty due to GVD is evaluated by considering Equation (33). Figure 11 shows the graphical representation of (33). It represents the power penalty Vs the fiber length for different bit rates (622 Mbps, $1 \mathrm{Gbps}$ and $2.5 \mathrm{Gbps})$. Similar to the first case, the power penalty in this case increases as the fiber length increases until the GVD reaches the point of the GVD noise floor, where the penalty tends to be infinity. The GVD noise floor is reached at a shorter fiber length as the bit rate increases. However, the GVD noise floor in this case is reached at longer fiber lengths. For example a $25 \mathrm{~km}$ fiber that imposes a $1 \mathrm{~dB}$ penalty can be used at a bit rate of $2.5 \mathrm{Gbps}$, which is longer than the fiber length that can be used at 2.5 Gpbs in the first case $(1.9 \mathrm{~km})$, which confirms the possibility of using the directly modulated small spectrumwidth source as an optical transmitter in the OANs as long as a bit rate of $2.5 \mathrm{Gbps}$ or less is specified.

\section{Power Penalty Due to GVD (An Externally Modulated Small Spectral-Width Source is Considered)}

In this case, the power penalty due to GVD is evaluated by considering Equation (35). Figures 12a-c shows the graphical representations of (35). They represent the power penalty Vs the fiber length for different bit rates (622 Mbps, $1 \mathrm{Gbps}$ and $2.5 \mathrm{Gbps}$ ). In this case, the performance is significantly improved because the GVD noise floors are reached at much longer fiber lengths compared with the GVD noise floors obtained in the first and second cases.

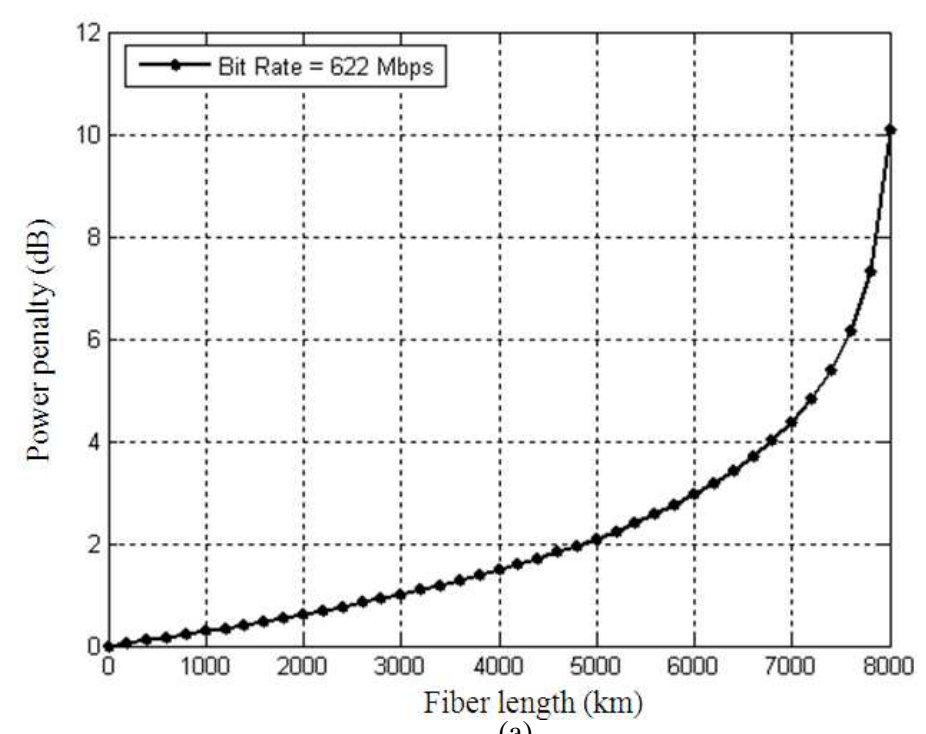

(a)

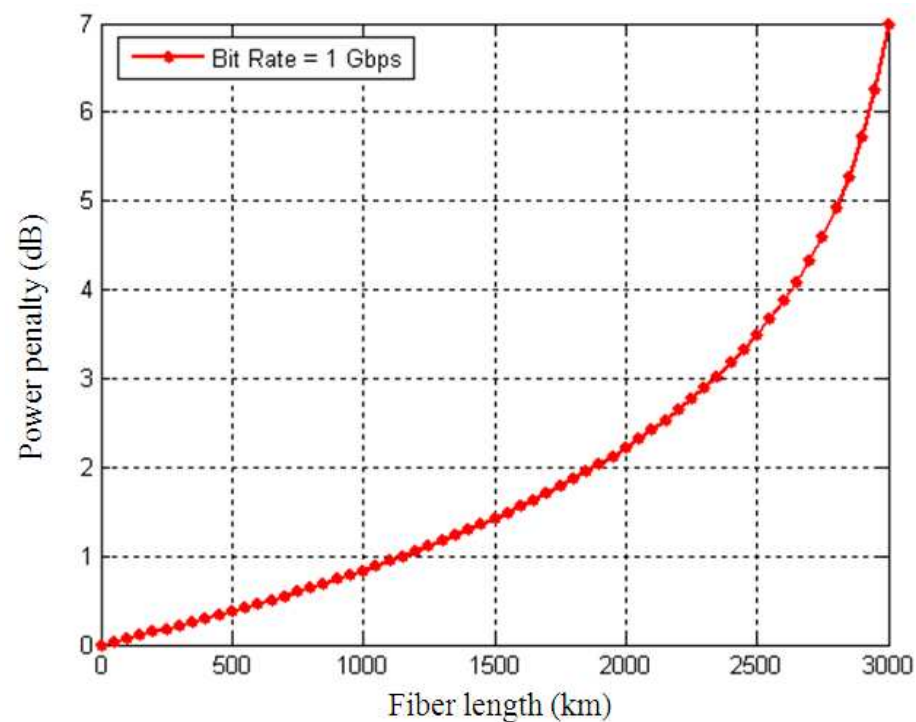

(b) 


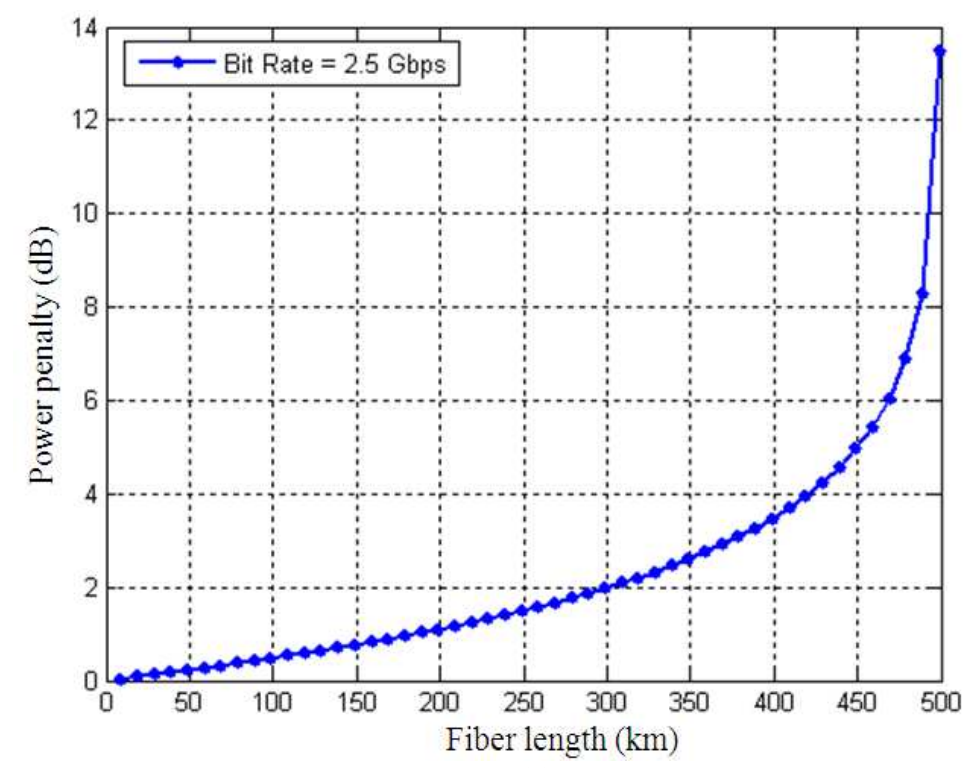

(c)

Fig. 12. (a) Power penalty due to GVD versus fiber length at $622 \mathrm{Mbps}$ (third case) (b) Power penalty due to GVD versus fiber length at 1 Gbps (third case) (c) Power penalty due to GVD versus fiber length at 2.5 Gbps (third case)

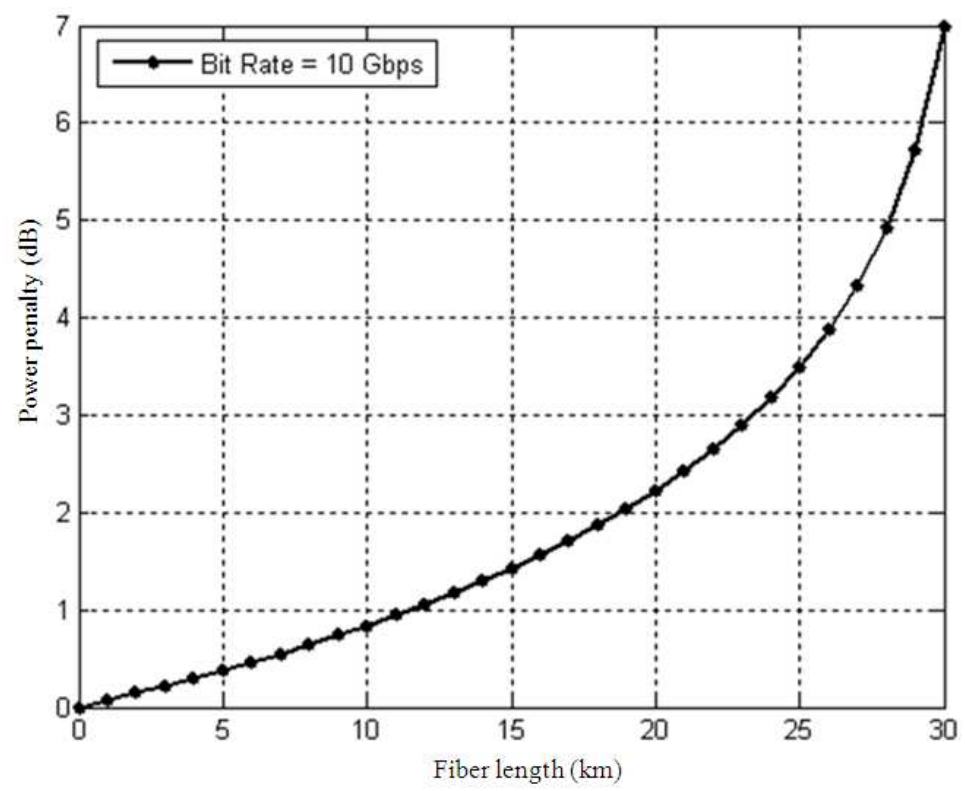

Fig. 13. Power penalty due to GVD versus fiber length at 10 Gbps (third case)

For example, the fiber lengths that can be used if a penalty of $1 \mathrm{~dB}$ is allowed are $2988.6 \mathrm{~km}, 1156.25 \mathrm{~km}$ and $185 \mathrm{~km}$ at $622 \mathrm{Mpbs}, 1 \mathrm{Gpbs}$ and $2.5 \mathrm{Gbps}$, respectively, which strongly confirms the possibility of using the externally modulated small spectrum-width source as an optical transmitter in the OANs where a minimum length of $20 \mathrm{~km}$ was specified. However, the allowed fiber length falls dramatically if the bit rate increases to 10 Gbps or more. Figure 13 shows the penalty versus the fiber length at $10 \mathrm{Gbps}$, where the GVD noise floor is reached at $31.25 \mathrm{~km}$. In this case a fiber length of $11.56 \mathrm{~km}$ can be used if a penalty of $1 \mathrm{~dB}$ is allowed, which necessitates the use of a dispersion management technique if a longer fiber length is required.

\section{Discussion}

In this study, we analyze the impact of in-band crosstalk, out-of-band crosstalk in an $\mathrm{N} \times \mathrm{N}$ AWG as they define and evaluate its performance as being used as 
multiplexer, demultiplexer, or router almost in all DWDMPONs and TDM/WDM-PONs. In addition to its usage as multiplexer, demultiplexer, or router, AWG has been involved and examined in many design concepts. In this context, Wang et al. (2006) applied an ArrayedWaveguide-Grating (AWG)-based multiport optical encoder/decoder (E/D) and Forward-Error-Correction (FEC) technique for the first time in an Optical CodeDivision Multiple-Access (OCDMA) system with high power contrast ratio between auto-/cross-correlation that can significantly suppress the interference noises in an asynchronous OCDMA system without using ultra long optical codes and optical thresh older (Wang et al., 2006). Yang presented an AWG-Based coder to generate codeword's from traditional maximum length sequences (m-sequence) in the Spectral Amplitude Coding (SAC) optical code-division multiple-access (OCDMA) network using two code keying, which led to achieve superior spectral efficiencies in the networks as compared to original m-sequences (Yang, 2010). Al-Junid et al. (2008) demonstrated an AWG-Based Encoder/Decoder (E/D) Module to generate and decode Zero Cross Correlation (ZCC) Code for Optical Code Division Multiple Access (OCDMA) systems (Al-Junid et al., 2008). Huang et al. (2010a; 2010b) Huang proposed an AWG-Based complete Complementary/Prime/Shifted Prime (CPS) code family which led to simplify the hardware implementation of encoder/decoder in the Optical Code-Division MultipleAccess (OCDMA) systems and eliminate the MultipleAccess Interference (MAI) via Spectral Amplitude Coding (SAC) OCDMA system under asynchronous transmission (Huang, et al., 2010a). Yen presented a Spectral Polarisation-Coding Optical Code-Division MultipleAccess (SPC-OCDMA) system for Radio-over-Fibre (RoF) transmissions by combining the quasi-orthogonality of Walsh-Hadamard codes with Arrayed Waveguide Grating (AWG) routers and a Polarisation Beam Splitter (PBS) in which the ability to encode-decode multiple WalshHadamard code words and cancellation of so called Multiple Access Interference (MAI) were achieved (Yen, 2012). Huang et al. (2010a; 2010b) proposed a cascaded dual-AWG-Based coder-decoder ( $\operatorname{codec}$ ) to reduce the crosstalk effect in Optical Code-Division Multiple Access (OCDMA) networks (Huang et al., 2010b).

\section{Conclusions and Future Work}

We have provided an analysis of different signal impairments that deteriorate the performance of TDM/WDM-PONs and lead to power penalties. First, we analyzed the impact of the in-band and out-of-band crosstalks. We concluded that in-band crosstalk has a stronger effect than out-of-band crosstalk because its noise floor is reached at a lower crosstalk noise level and with fewer crosstalk components. We found that the inband crosstalk should be kept below $-37 \mathrm{~dB}$ and $-34 \mathrm{~dB}$ to maintain the power penalty at less than $1 \mathrm{~dB}$ if 15 and 7 in-band crosstalk components are considered, respectively. The out-of-band crosstalk should be kept below $-20.3 \mathrm{~dB}$ and $-17.18 \mathrm{~dB}$, to maintain the power penalty at less than $1 \mathrm{~dB}$ if 240 and 56 out-of-band crosstalk components are considered. Secondly, we analyzed the impact of the GVD. It was observed that the GVD noise floor is reached at a shorter fiber length as the bit rate increases where the penalty tends to infinity. It was concluded that a significant improvement in which the GVD noise floor is reached at longer fiber can be achieved if an externally modulated, small spectralwidth source is used when a bit rate of $622 \mathrm{Mbps}, 1 \mathrm{Gps}$, or $2.5 \mathrm{Gbps}$ is used. However, a dispersion management technique becomes necessary if the bit rate increases to 10 Gbps or more. Future research planned on this topic concerning the first stage of the design in which the performance of the proposed TDM/DWDM optical access network architecture will be evaluated based on mathematical analyses. In those mathematical analyses, the BER will be calculated based on Signal-to-Noise Ratio (SNR) evaluation at the receiver. This involves tracing the path of the digital signals along the way between the transmitter and the receiver considering effects on it at each stage. The SNR will be estimated by calculating both, the signal and noise power at the receiver. A further research planned on this topic concerning the second stage of the design where the EDFA is intended to be incorporated after being optimized based on analytical and simulation models in terms of its Power pump (Pp), Erbium-Doped Fiber (EDF) length, overlap factor, erbium ions concentration and configuration used, which could lead to improve the design and reduce the cost.

\section{Acknowledgement}

Authors would like to convey their acknowledgement to the department of electrical electronic and systems engineering in the national university of Malaysia UKM for their help and support.

\section{Author's Contributions}

Ibrahim Mohamed: As the main author of the manuscript, he came up with the proposed solution of long-reach optical access as well as the corresponding methodology that should be followed in the development process, which mentioned in the introductory part of the manuscript.

Mohammad Syuhaimi Ab-Rahman: As the co author of the manuscript, he provided detailed solution that should be followed in step by step approach in order to accomplish the proposed solution. Both authors have 
contributed in preparation, development and writing of the manuscript.

\section{Ethics}

This article is original and contains unpublished material. The corresponding author confirms that all of the other authors have read and approved the manuscript and no ethical issues involved.

\section{References}

Agrawal, G.P., 2005. Lightwave Technology: Telecommunication Systems. 1st Edn., John Wiley and Sons, Hoboken, ISBN-10: 0471741396, pp: 480.

Agrawal, G.P., 2010. Fiber-Optic Communication Systems. 4th Edn., John Wiley and Sons, New York, ISBN-10: 0470918519, pp: 626.

Al-Junid, S., S. Shaari, S.A. Aljunid and M.S. Anuar, 2008. Design of encoder and decoder module for Optical Code Division Multiple Access (OCDMA) Systems for Zero Cross Correlation (ZCC) code based on Arrayed Waveguide Gratings (AWGs). Proceedings of the International Conference on Electronic Design, Dec. 1-3, IEEE Xplore press, Penang, pp: 1-4. DOI: 10.1109/ICED.2008.4786725

Grobe, K. and J.P. Elbers, 2008. PON in adolescence: From TDMA to WDM-PON. IEEE Commun. Magazine, 46: 26-34. DOI: 10.1109/MCOM.2008.4427227

Huang, J.F., C.T. Yen and Y.W. Tu. 2010b. Reduction of linear crosstalk over waveguide-grating-based optical CDMA coders/decoders. J. Optical Commun. Network., 2: 975-983. DOI: 10.1364/JOCN.2.000975

Huang, J.F., D.J. Nieh and C.C. Yang. 2010a. Multiple interference suppression with CPS coding Scheme over Asynchronous Optical CDMA System. Proceeding of the International Conference on Communication Systems, Nov. 17-19, Singapore, IEEE Xplore press, pp: 189-193.

DOI: 10.1109/ICCS.2010.5686391

Inoue, Y., Y. Ohmori, M. Kawachi, S. Ando and T. Sawada et al., 1994. Polarization mode converter with polyimide half waveplate in silica-based planar lightwave circuits. IEEE Photon. Technol. Lett., 6: 626-628. DOI: 10.1109/68.285561
Kaneko, A., T. Goh, H. Yamada, T. Tanaka and I. Ogawa, 1999. Design and applications of silica-based planar lightwave circuits. IEEE J. Selected Topics Quantum Electron., 5: 1227-1236. DOI: $10.1109 / 2944.806745$

Kani, J.I., 2010. Enabling technologies for future scalable and flexible WDM-PON and WDM/TDMPON systems. IEEE J. Selected Topics Quantum Electron., 16: 1290-1297. DOI: $10.1109 /$ JSTQE.2009.2035640

Kazovsky, L.G., S.W. Wong, V. Gudla and P.T. Afshar, 2011. Challenges in next-generation optical access networks: Addressing reach extension and security weaknesses. IET Optoelectron, 5: 133-143.

DOI: 10.1049/iet-opt.2011.0027

Smit, M.K., 1988. New focusing and dispersive planar component based on an optical phased array. Electron. Lett., 24: 385-386.

DOI: $10.1049 / \mathrm{el}: 19880260$

Syuhaimi, M. and I. Mohamed, 2013. Highly utilized fiber plant with extended reach and high splitting ratio based on AWG and EDFA characteristics. ETRI J., 35: 786-796 DOI: 10.4218/etrij.13.0112.0793.

Takahashi, H., Y. Hibino, Y. Ohmori and M. Kawachi, 1993. Polarization-insensitive arrayed-waveguide wavelength multiplexer with birefringence compensating film. IEEE Photonics Technol. Lett., 5: 707-709. DOI: $10.1109 / 68.219718$

Wang, X., N. Wada, G. Cincotti, T. Miyazaki and K. Kitayama, 2006. Demonstration of over $128-\mathrm{Gb} / \mathrm{s}-$ capacity (12-User 10.71-Gb/s/User) asynchronous OCDMA using FEC and AWG-based multiport optical encoder/decoders. Photonic Technol. Lett., 18: 1603-1605. DOI: 10.1109/LPT.2006.879594

Yang, C.C., 2010. Spectral efficiencies of the optical CDMA-based PONs using two-code keying. IEEE Commun. Lett. 14: 767-769. DOI: 10.1109/LCOMM.2010.08.100585

Yen, C.T., 2012. Optical code-division multiple-access embedded with a polarisation diversity scheme for radio-over-fibre transmissions. IET Optoelectro., 6: 131-139. DOI: 10.1049/iet-opt.2010.0074 